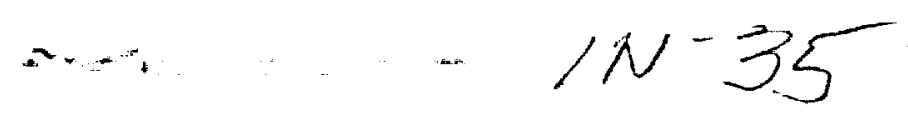

NASA Technicàl Memorandum 106596

AIAA-94-2985

12654
$13 P$

\title{
Instrumentation for In-Flight SSME Rocket Engine Plume Spectroscopy
}

George C. Madzsar

Lewis Research Center

Cleveland, Ohio

Randall L. Bickford

Aerojet Propulsion Division

Sacramento, California

and

(NASA-TM-106596) INSTRUMENTATION

N94-35268

David B. Duncan FOR IN-FLIGHT SSME ROCKET ENGINE

Duncan Technologies PLUME SPECTROSCOPY (NASA. LEWIS Research (enter) $13 \mathrm{p}$

Unclas

Auburn, California

G3/35 0012654

Prepared for the

30th Joint Propulsion Conference

cosponsored by AIAA, ASME, SAE, and ASEE

Indianapolis, Indiana, June 27-29, 1994

National Aeronautics and Space Administration 


\title{
INSTRUMENTATION FOR IN-FLIGHT SSME ROCKET ENGINE PLUME SPECTROSCOPY
}

\author{
George C. Madzsar \\ National Aeronautics and Space Administration \\ Lewis Research Center \\ Cleveland, Ohio 44135 \\ Randall L. Bickford \\ Aerojet Propulsion Division \\ Sacramento, California 95813 \\ David B. Duncan \\ Duncan Technologies \\ Auburn, California 95603
}

\begin{abstract}
This paper describes instrumentation that is under development for an in-flight demonstration of a plume spectroscopy system on the space shuttle main engine. The instrumentation consists of a nozzle mounted optical probe for observation of the plume, and a spectrometer for identification and quantification of plume content. This instrumentation, which is intended for use as a diagnostic tool to detect wear and incipient failure in rocket engines, will be validated by a hardware demonstration on the Technology Test Bed engine at the Marshall Space Flight Center.
\end{abstract}

\section{INTRODUCTION}

Integrated vehicle health management (IVHM) has been identified by the NASA Office of Space Flight as the highest priority technology for present and future space transportation systems1. Systems designed for IVHM will efficiently perform checkout, test, and monitoring of space transportation vehicles and subsystems before, during, and after operation. The primary purpose of IVHM is to increase safety and reliability while simultaneously reducing costs. NASA plume spectroscopy research and development efforts over the past 8 years have been oriented towards the goals of (1) detecting the onset of severe erosion during flight or test and (2) identifying degraded components for postflight maintenance. These goals offer significant safety enhancement and operations cost reductions for reusable systems such as the Space Transportation System.

Hardware for an in-flight plume spectroscopy system consists of an optical probe to collect light from the plume, a fiber-optic cable to transmit the light to a spectrometer which discriminates the species in the plume, and a data recording system. Specific devices under development include the nozzle mounted optic assembly, and the Fabry-Perot interferometric (FPI) spectrometer. The probe, which is mounted to the aft manifold of the engine nozzle, collects the spectral radiation; and the FPI spectrometer filters this plume radiation and measures the intensity at wavelengths that are characteristic of the eroding materials. Although this hardware is specifically intended for use on the space shuttle main engine (SSME), the technology is generic, and can readily be incorporated onto other engines. This hardware is scheduled for testing on the Technology Test Bed (TTB) engine at the Marshall Space Flight Center (MSFC).

Capabilities of a flight plume spectroscopy system will include real time measurement and analysis of material erosion for engine safety monitoring, determination of performance parameters for feedback into the engine controller, and recording high-resolution, high-speed spectral data for post firing analysis of the engine.

\section{BACKGROUND}

As rocket engine components experience wear or degradation, eroded materials may be entrained in the plume. Visible plume anomalies often precede many engine failures $^{2-4}$, e.g., the flashes of green observed in the plumes from engines using copper lined combustion chambers. These plume anomalies typically result from eroded materials passing through the engine and reacting with the propellants in the combustion chamber. The distinct color changes in the plume correspond to the frequency of light emitted by excited atoms in the hot exhaust.

Spectroscopic techniques are routinely used for laboratory analysis of the elemental composition of liquid and solid materials. Plume spectroscopy makes use of optical spectroscopic techniques to study the spectral radiation emitted or absorbed by the exhaust gases. The identification and quantification of the elemental species in the plume can be used to assess the health, safety, and maintenance needs of an engine. Plume spectroscopy is a nonintrusive, multiparameter measurement technique for engine health, safety, and maintenance management. 
Most critical engine components are in contact with the propellants. Material loss due to wear, component distress, or component breakage results in particulates passing through the engine and into the combustion chamber (carried by the propellants) where they produce spectrally bright emissions in the plume. Measurements of these spectral emissions provide insight into the type(s) and amount(s) of eroded material.

Plume emissions can be converted to engineering information by the three step process illustrated in Fig. 1. First, spectral emissions from the exhaust plume are collected using an optical probe mounted on the engine nozzle. Second, the elemental constituents of the plume are identified and quantified using a spectrometer. Finally, eroding alloys and engine components are identified and quantified using numerical algorithms that assess metal concentrations and determine component of origin.

Spectral emissions from the plume of the SSME are routinely monitored during ground testing and have been successfully correlated to component wear. Spectral databases exist for the TTB and from flight-certification firings, at NASA Stennis Space Center (SSC), of the SSME. Spectroscopic techniques are used in all postfiring reviews: it is used by spectroscopists to develop time histories of engine erosion, to aid in the identification of worn engine components, and to identify components that need further evaluation prior to the next engine firing. However, the spectral history during SSME flight is missing. The intent of the instrumentation described in this paper is to extend this technology to flight.

Figures 2 and 3 display time slice spectral histories recorded from TTB engine firings 017 and 024 . These figures illustrate the detection of engine degradation utilizing plume spectroscopy. The spectra in Fig. 2 correspond to a nominal engine firing, with emissions from the hydroxyl radical $(\mathrm{OH})$ and broadband background consistent over time. The $\mathrm{OH}$ and background emissions are always observed in the SSME plume with reasonably constant and consistent intensities. The spectra of Fig. 3 are from an engine as it is degrading. In addition to the normal $\mathrm{OH}$ and background, numerous line emissions from various metallic species can be seen. Note that intensity of the $\mathrm{OH}$ and background in this figure remain constant over time, however emissions from metallic species (specifically the chromium lines at approximately 424 nanometers $(\mathrm{nm})$ ) vary with time. Since intensity is a function of number density, line intensities are proportional to the amount of material that is eroding from the engine component. Analysis of the TTB engine after firing 024 revealed that the faceplate of the engine had eroded. This faceplate is fabricated from the alloy Inconel 625 , which contains chromium.

\section{THEORY}

Plume spectroscopy can be performed using emission or absorption techniques. Emission spectroscopy is based on measurement of emissions radiating from excited species, and absorption spectroscopy is based on the absorption of light by the constituents of the plume.

For emission spectroscopy, intensity is proportional to the number density of the emitting species. It is assumed that the atoms in the emitting system are in thermal equilibrium, there is a low population density of the emitting atoms, and that the emitted light is not reabsorbed. The intensity of an atomic line is given by the equation:

$$
I=\frac{A_{i j} N h(v) g_{i} e^{-E i / k T}}{Z(t)}
$$

Where $I$ is the emission intensity, $A_{i j}$ is the Einstein transition probability for spontaneous emission, $\mathrm{N}$ is the number density, $h$ is the Planck constant, $v$ is the frequency, $g_{i}$ is the statistical weight for each sublevel with an energy of $E i$, $\mathrm{k}$ is the Boltzmann constant, $\mathrm{T}$ is the absolute temperature, and $Z(t)$ is the partition function. Note that at temperatures approaching $3300 \mathrm{~K}$ (the maximum temperature expected in the hot regions of the SSME plume), $\mathrm{Z}(\mathrm{t})$ is approximately $g_{0}$, where $g_{0}$ is the statistical weight of the ground state. In these high temperature regions, a sufficient concentrations of free electrons are available for electron impact excitation of metal atoms. Virtually the entire population of atoms and molecules in excited states occurs through electron impact.

The SSME combustion chamber operates at 205 atmospheres during $109 \%$ power conditions with an oxidizer to fuel ratio of $6: 1$. Stagnation temperature in the combustion chamber is approximately $3300 \mathrm{~K}$, and $3200 \mathrm{~K}$ in the Mach disc. The engine is overexpanded at sea level and underexpanded in the vacuum of space. During engine start and shutdown transients, the nozzle undergoes significant distortion and is subjected to thermal, mechanical, and pneumatic shock. From video recordings the nozzle has been observed to distort approximately $150 \mathrm{~mm}$. Published vibration criteria indicates that the nozzle experiences a $7500 \mathrm{~g}^{\text {shock }}{ }^{5}$. The acoustic environment at the SSME nozzle exit plane is 130 to $170 \mathrm{~dB}$. In addition, pressure and temperature spikes pass through the nozzle due to the movement of the normal shock wave. Since this shock wave moves outward from inside to outside the nozzle during engine start, the pressure at the exit plane exhibits a spike as the shock wave passes. A similar pressure spike exists during engine shut-down since the normal shock wave will move back into the nozzle.

Results from the Two Dimensional Kinetics(TDK) $\operatorname{code}^{6}$ indicate a convective heat flux of $2.7 \mathrm{MW} / \mathrm{m}^{2}$, a static temperature of $1430 \mathrm{~K}$, and static pressure of 0.4 atmospheres near the nozzle wall at the exit plane. However, measurements during TTB testing indicate steady state static temperature and pressure of $670 \mathrm{~K}$ and 0.2 atmospheres, respectively. During design of the probes, the more hostile environment predicted by TDK was assumed, rather than the measured results. 


\section{NOZZLE MOUNTED OPTICS}

An optical system, which is mounted to the aft manifold of an engine nozzle, can be used to observe spectral phenomena emanating from, or absorbed by the plume. The following discussion relates the application of nozzle mounted optics specifically to the SSME.

EMISSION SOURCE The primary consideration for nozzle mounted optics is the view of the exhaust gases. The Mach discs, combustion chamber, and throat are areas of strong emissions and therefore logical sources for emission measurements; the lower temperature and pressure conditions at the nozzle exit plane makes it suitable for absorption measurements. Other considerations include; spectral characteristics of the plume constituents, thermal and structural dynamics of the engine, and flight related issues such as optic size, weight, and mounting.

To monitor plume spectra during flight the probe can be (1) mounted on the nozzle to view down at the Mach disc; (2) mounted on the nozzle to view across the exit plane; (3) mounted on the lip of the nozzle to view up into the nozzle; (4) mounted off the engine (on the vehicle) to view the Mach disc; (5) mounted in the side of the combustion chamber or throat to view the chamber; or (6) mounted in the ignitor or injector to view the chamber and throat. Due to engine gimbaling during ascent, option 4 is not viable. For this effort, only nozzle mounted optics were considered since minimal engine modifications are required to retrofit them into existing engine systems.

The three types of optics under development consist of a forward facing optic that collects emissions emanating from within the engines' combustion chamber and throat; an aft facing optic that collects emissions emanating from the Mach disc; and an optic to measure absorption across the exit plane of the nozzle. Figure 4 schematically shows the probe, views, and spectrometer in relation to the engine.

The probes can be mounted on the SSME aft manifold using existing holes provided for the side load arrestor mechanism (SLAM) ring mounts. These holes, which are found on all existing SSME nozzles, are used during aspirated diffuser testing at SSC and are not used otherwise. The holes are plugged with bolts during ambient firings and flight operation. The nozzle mounted optic will mount on studs fabricated to match the geometry of the existing bolts used to fill these holes. Analysis and test verification has demonstrated that the these probes have no effect on the engine or nozzle in terms of mass loading, thermal loading, or mechanical effects and does not decrease engine life or safety. An inherent advantage of nozzle mounted optics is that in case of structural failure, fragmented optics pieces will not cause damage to the engine since the fragments will fall below the engine.
CHAMBER VIEWING OPTIC A schematic of this optic is shown in Figure 5. Spectral emissions emanating from the chamber/throat are focused into an optical fiber, which transmits the light to the spectrometer. This view eliminates the need for subtracting the solar background. Calculations have shown that chamber radiation is orders of magnitude greater in intensity than Mach disc radiation, allowing for measurement of lower level signals while increasing the signal to noise ratio. TTB testing of this optic has demonstrated survivability for over 10 firings and 1000 seconds of engine operation.

A lens/turning optic focuses and directs the emissions. The lens defines the field of view (FOV), and the turning optic directs the light into the optical fiber. The turning optic is required since the radius of curvature of the aft manifold exceeds the allowable bend radius of the optical fiber. To simplify the optical design and minimize optical losses, the f-number matching lens and turning optic are integrated into a single element. The FOV required to observe the throat from the nozzle lip is 4.1 degrees. Since the numerical aperture of a fused silica optical fiber is approximately 0.28 in the ultraviolet (UV) (which is roughly equivalent to 30 degrees acceptance angle), a lens is utilized to match the viewing angle with the acceptance angle. Using a $2.5 \mathrm{~mm}$ lens, the throat image spot size is $0.4 \mathrm{~mm}$. When focused on a $0.8 \mathrm{~mm}$ diameter optical fiber, a FOV twice the throat area is provided, enabling observation of the throat during all aspects of engine firing; including nozzle distortion during the start/stop transient.

The design for the chamber viewing optic is a compromise between size, weight, and strength. The optic, which weighs 0.25 Lbs., is fabricated from precipitation hardened zirconium-copper with a zirconium oxide thermal barrier coating applied to the flowfield exposed end. Since the engine is overexpanded at sea level, the barrel shock extends from the nozzle lip to the downstream Mach disc. The optic is located outside the boundary of the barrel shock to avoid shock impingement. At high altitude, the engine is underexpanded and the barrel shock will be very weak or non-existent with low or no back pressure (vacuum). Since the optics will be located outside the barrel shock boundary, convective heating during sea level operation is the worst case thermal environment affecting survivability. Conduction from the optic into the aft manifold (through which liquid hydrogen circulates) is used to cool the device.

A chamber viewing optic has been tested on the TTB engine. The observed spectra appears similar to that of a black body radiation source with the intensity peak at approximately $520 \mathrm{~nm}$ and no atomic line structure. Several mechanisms have been postulated to explain these results; among them blackbody radiation from high pressure gases, Rayleigh scattering off molecules within the chamber, and scattering off the chamber wall. Additional test are planned with a modified chamber viewing optic. A new lens will be fabricated to change the field of view to just below the throat. The pres- 
sure in the nozzle drops off significantly below the engine throat. It is thought that observing the plume in a lower pressure regime may enable observation of spectral lines.

MACH DISC VIEWING OPTIC The Mach disc is readily accessible and provides a good optical signal. Under the Optical Plume Anomaly Detection ${ }^{7}$ (OPAD) and Diagnostic Test Facility ${ }^{8}$ (DTF) programs, emissions from the Mach discs are measured. Figure 6 displays a schematic for this device. This optic is basically a truncated version of the chamber viewing optic. The primary difference is the elimination of the turning prism and its associated structure that overhangs the aft manifold to look up into the chamber. At this time, finite element (FE) structural and thermal analyses for this device have not been performed. However, since the design for this device is similar to the chamber viewing optic, it is anticipated that this device will not experience undue stress. Also, the shortened probe will experience lower bending stresses than the chamber viewing probe. Since this device is located outside the flowfield boundary thermal loads will be minimal. Based on video tapes of the nozzle, condensation and freezing of optical surfaces will likely be the principal thermal issue due to thermal contact with the liquid hydrogen aft manifold. A nitrogen purge has been incorporated into the design of this probe to prevent condensation and freezing.

Note that the Mach disc may not be the ideal source for plume measurements on a flight system. Radiation from sources behind the discs (e.g., the Sun or plumes from other engines) may enter the collection optics along with the desired plume emissions, requiring that the intensity of these potentially varying radiation sources be known to enable their removal. Other difficulties include movement of the Mach discs with respect to the nozzle (since disc location is a function of atmospheric pressure), the disappearance of the Mach discs in the vacuum of space, and that not all gases (and, therefore, not all particulates) pass through the Mach discs.

ABSORPTION OPTIC Figure 7 shows a schematic of this device. The Nozzle Mounted Absorption Optic (NMAO), when used in a matched pair, propagates light through the exit plane flowfield of the SSME enabling absorption spectroscopy. Absorption spectroscopy allows for a more easily quantifiable measurement of flowfield entrained metal concentrations than does emission spectroscopy. The NMAO collimates light emitted from a fiberoptic cable into a beam that passes through the SSME exit plane. The transmitted signal is collected using a second NMAO. Both optical assemblies are bolted to the SSME aft manifold.

Considerations for the NMAO include aperture sizing, throughput requirements, FOV, and other optical characteristics. The system is sized to allow the optical source and detector systems to be mounted remote from the engine. The NMAO sizing was based on the throughput requirements to perform narrow line atomic absorption spectroscopy with a hollow cathode lamp source.
A tradeoff exists for signal-to-noise ( $\mathrm{S} / \mathrm{N})$ and alignment sensitivity. As the beam expands, the signal-to-noise decreases because the light arriving at the detector decreases. A larger optic aperture improves the $\mathrm{S} / \mathrm{N}$ by collecting more of the transmitted signal. The upper limit on aperture size is governed by weight and interaction with the flowfield gases. The relationship between the beam spot size on the far side of the nozzle, collected light, and aperture were evaluated. A nominal $18 \mathrm{~mm}$ aperture was selected based on available offaxis parabolic mirrors, probe weight and size.

As stated earlier, the nozzle distorts approximately 150 mm during the start/stop transient. It causes a $315 \mathrm{~mm}$ shift in the FOV, requiring a $630 \mathrm{~mm}$ diameter FOV to accommodate this distortion. At mainstage, nozzle deflection is minimal allowing a relatively small FOV to be utilized. A nominal beam diameter of $80 \mathrm{~mm}$ was chosen, providing allowance for vibration and nozzle distortion without excessively penalizing light throughput. The beam diameter is adjustable by controlling the position of the fiberoptic cable termination.

The NMAO is located outside the flowfield boundary so thermal loads will be minimal. Based on video tapes of the nozzle, condensation and freezing of optical surfaces will likely be the principal thermal issue due to thermal contact with the liquid hydrogen aft manifold. A nitrogen purge has been incorporated into the design of this probe to prevent condensation and freezing.

\section{SPECTROMETER}

Spectral characteristics considered with respect to the spectrometer include the relative amounts of atomic and molecular particulates in the plume, and the wavelengths of their spectral phenomena. For a hydrogen-oxygen engine such as the SSME, spectral activity of interest occurs between 300 and $1000 \mathrm{~nm}$. Spectra from metallics occur between 300 and $500 \mathrm{~nm}$; emissions from the hydroxyl radical occur between 305 and $320 \mathrm{~nm}$; and $\mathrm{H}_{2} \mathrm{O}$ activity occurs between $700-1000$ nm.

The number and identities of the species to be monitored is based on the requirement to monitor all critical engine components. Evaluation of the alloy and elemental content of the various components in the propellant flow paths reveals a minimum number of species that must be monitored. The SSME alloys, their elemental constituents, and the weight fraction of these constituents are listed in Ref. 9. From this list it is evident that one or more of the elements chromium, cobalt, iron, nickel, manganese, and tungsten are constituents in each alloy. In addition, copper and calcium are elemental constituent of critical SSME components; e.g. calcium in the armalon cages of the turbopump bearings, and copper in the combustion chamber liner. Therefore, monitoring for these eight 
species enables detection of erosion from all SSME alloys and critical components.

Using the techniques described in Ref. 4, radiant power values can be calculated for the eight elemental species at SSME conditions. From these values and from values for spectrometer sensitivity, minimal detectability limits (MDL's), in parts per million and grams per second, can be derived. Reference 9 lists the species, their emission wavelengths and atomic line widths, and the calculated fluxes arriving at an optical collector located on the lip of the SSME nozzle for each atomic species at MDL concentrations. Alloy loss rate MDL's can be calculated from the specie MDL's.

When a single alloy is present in the plume, the identity of its elemental constituents and, therefore, of the alloy itself can readily be determined. If multiple alloys are present in the plume, the identification process is, again, relatively simple if each alloy is composed of different elements. However, many SSME alloys contain some of the same elemental species, the differences being various weight fractions of the elements. If several alloys are present in a plume and if these alloys contain some of the same elemental constituents, the spectra of the individual elements are summed, and a direct determination of the identities and amounts of the alloys cannot be made solely from spectroscopic measurements. However, from knowledge of which alloys may be present in the plume, along with knowledge of constituents content, weight fraction, element spectral characteristics and knowledge of the temperature at the location of emission, algorithms consisting of a series of simultaneous equations can be developed and solved10,11. The output of these algorithms yields the identities and amounts of alloys in the plume.

An interferometer based spectrometer was selected for this application based on its small size, high-resolution, and high-speed spectral acquisition capabilities. A Fabry-Perot interferometer was selected based on its spectral bandwidth, simplicity of design, and commercial availability. Engineering an appropriate housing for the interferometer resulted in a rugged, compact, lightweight spectrometer.

An FPI is a tunable narrow band filter (tunable etalon), which produces a periodic set of transmission peaks (orders) corresponding to wavelengths that are integer multiples of the mirror spacing. For spectroscopic purposes, the radiation passing through the FPI requires additional filtering to reduce the number of orders transmitted to the detectors. Dielectric narrow bandpass interference filters are used for this order sorting. The interferometer allows radiation at a finite number of wavelengths to pass through (seen as the transmission peaks). The wavelength of one of the transmission peaks corresponds to the emission wavelength of a specie of interest. Exiting the interferometer, radiation at the 'unwanted' wavelengths are eliminated by a bandpass filter. The tunable interferometer in combination with the fixed bandpass filter yields a high resolution spectrometer ${ }^{9}$.
The wavelengths for the eight species to be monitored lie between 320 and $430 \mathrm{~nm}$, dictating the bandwidth of the spectrometer. Spectrometer resolution must be sufficient to resolve closely spaced and narrow spectral lines. The narrowest of the 8 spectral lines is $0.2 \mathrm{~nm}$ wide ${ }^{9}$. Since the spectrometer is a scanning and data sampling device, sampling theory must be considered. Nyquist sampling theory dictates that as a minimum, two points must be measured (corresponding to the peak and adjacent valley) to resolve a waveform and prevent aliasing. Typically, more than two points are used to insure proper resolution; for the spectrometer four points will be used, corresponding to a spectrometer wavelength resolution of $0.05 \mathrm{~nm}$.

The etalon mirror gap controls the spacing between transmission maximums. For broadband input, smaller gaps increase the transmission peak spacing and larger gaps decrease it. The mirror spacing controls how many orders lie within the bandpass filter transmission bandwidth. The free spectral range (FSR) that is less than the filter bandwidth allows transmission from more than one order which is detrimental when monitoring for an atomic specie emission. Operating with a FSR larger than the filter bandwidth results in excessive FPI bandwidth, thereby increasing the background transmission. Background is minimized by selecting a FSR approximately equal to the filter bandwidth.

Finesse, which is the ratio of the transmission bandwidth to the spacing between orders (FSR), determines the FPI background transmittance at resonance. Increasing finesse decreases the transmission bandwidth with a fixed mirror gap. Off resonance transmission contributes to background signal.

A breadboard FPI spectrometer has been fabricated and tested. This 3 channel unit ( $324.7 \mathrm{~nm}, 377.6 \mathrm{~nm}, 425.4 \mathrm{~nm}$ ) was tested using a seeded flame (copper and chromium) as the optic "source". The copper line at $324 \mathrm{~nm}$ and the chromium line at $424 \mathrm{~nm}$ were selected for this unit since they have been identified as two of the species required for SSME monitoring, and the wavelengths are at the extremes of the bandwidth. The thallium line at $377.6 \mathrm{~nm}$ was included in this unit since it does not appear in the SSME and could therefore be used as wavelength reference. Laboratory testing of this device demonstrated the viability of FPI spectrometers. A six-channel unit has subsequently been successfully tested on the SSC Diagnostic Test Facility engine. Currently, an eight-channel unit (using a flight-type optical design) is being fabricated for testing on the TTB engine.

The intent of the 3 channel unit was to: (1) validate the concept, (2) validate the design approach, and (3) evaluate the performance of FPI based spectrometers. Testing was performed to evaluate the optical sensitivity of the unit, and evaluate the effects of vibration, static acceleration, temperature variations, and background radiation on the overall performance. 
Using a seeded burner rig as the optical source, it was found that the measured signals recorded during the tests agreed with the calculated signal strength, thereby verifying the predicted sensitivity of the unit. Vibrational testing was performed by observing the detector output while subjecting the FPI spectrometer to random impacts of varying amplitudes. Minimal changes were observed during the impacts. Static acceleration tests were performed by rotating the orientation of the spectrometer with respect to the earth and measuring the spectrometer's finesse at the various orientations. It was found that the finesse does vary with orientation, thereby indicating sensitivity to static acceleration. This sensitivity to acceleration was not great enough to warrant the modification and/or redesign of the spectrometer. To test for sensitivity to temperature variations, the finesse was measured during heating of the spectrometer with a heat gun. It was found that thermal induced drift does occur. This was not unexpected, since the interferometer itself has a long path length and large diameter ( 1 inch) optics. Smaller diameter optics and a shorter path length interferometer will minimize thermal effects. To test for sensitivity to background radiation, the cover of the spectrometer was removed to allow leakage of ambient light. It was found that the spectrometer can be extremely sensitive to light leakage.

From the tests it was determined that a unique reference specie was not necessary. Instead, a HCL containing the 8 species identified earlier will be used, resulting in an accurate wavelength reference and calibration point for all 8 species. Photomultiplier tubes (PMT's) will be used in place of silicon detectors for the 8 channel unit. The PMT's have greater sensitivity and dynamic range in the 300 to $500 \mathrm{~nm}$ bandwidth. Their responsivity falls off appreciably above $650 \mathrm{~nm}$; resulting in an increased measurable range of spectral intensities in the bandwidth of interest while decreasing the unit's sensitivity to infrared $(\mathbb{R})$ radiation.

The FPI spectrometer that is currently being fabricated is shown schematically in Fig. 8. Spectral radiation enters from the optical fiber and passes through a lens where it is collimated. The collimated light then proceeds through a beam splitter, which discards the IR and UV radiation that is not of interest. The beam splitter also enables introduction of spectral radiation from a hollow cathode lamp ( $\mathrm{HCL}$ ) into the device for calibration purposes. The radiation then passes through the tunable etalon. The etalon mirror spacing is controlled by piezoelectric drivers. A dielectric coating was placed on the etalon mirrors allowing operation from 320 to $430 \mathrm{~nm}$, with a reflectivity of approximately $93 \%$. Outside of the operating range, transmission through the FPI falls off substantially, thereby further blocking unwanted UV radiation from the $\mathrm{OH}$ emissions, and unwanted IR radiation from the $\mathrm{H}_{2} \mathrm{O}$ molecules. Blockage of unwanted radiation helps ensure a high signal to noise ratio. Exiting the etalon, the light enters an order sorting filter/prism/beam splitter, that splits off the 'order' containing the wavelength of interest (by means of interference filters coated onto the prism surface) and directs the spectral radiation into a photomultiplier tube. In essence, the interference filters place a $1 \mathrm{~nm}$. wide 'window' around the wavelength corresponding to specie emission, and the tunable etalon steps through that window in $0.05 \mathrm{~nm}$ increments.

The photomultiplier tubes selected for the FPI have detection thresholds of $10^{-12}$ watts. Testing has shown that optical losses in the system will attenuate the incoming signal by a factor of 100 . From these values, it has been determined that a spectrometer entrance aperture greater than $0.2 \mathrm{~mm}$ diameter will provide an optical signal that exceeds the photomultiplier detection limit at the SSME MDL, considering losses.

The complete spectrometer system consists of the FPI spectrometer, the HCL, an associated processor module for control, data acquisition, data storage, and processor electronics. The FPI software consists of pre-test processing, run time processing, post test processing, and input/output (I/O) interface routines. Pre test processing performs the initialization and set up of the system. This includes self calibration (using the HCL) and self test of the data acquisition/processing electronics to insure its proper operation. During engine firing, the run time processing routine will generate the FPI scan waveform for the piezoelectric drivers, perform the background subtraction of the species, and check spectral intensity against specie redlines. Data acquisition, background subtraction, and redline check occurs simultaneously for the 8 channels every 10 milliseconds.

At the conclusion of the engine firing the post test processing routines will calculate and check spectrometer finesse, and replay stored spectral data. Human interaction with the system will be through a graphical user interface that enables input of user defined configurations, and values for specie redlines.

Signal processing is required to extract the atomic lines and identify the alloy/component from line information. The line radiation must be extracted from the overall radiation at a given wavelength. The overall radiation is the sum of emissions from the various atomic sources, molecular sources, and background chemiluminescence. The FPI spectrometer has an active background subtraction capability. As the intensity is measured at $0.05 \mathrm{~nm}$ increments in the $1 \mathrm{~nm}$ window around each spectral line, sample and hold circuitry is used to measure the minimum and maximum values. The maximum intensity value corresponds to the spectral line peak, and the minimum intensity value (intensities measured on either 'side' of the spectral line) corresponds to the background. Subtraction of the minimum from the maximum reveals the absolute intensity of the spectral line.

The entire system is controlled by a commercially available 386 based computer. The computer has a 2 channel 12-bit digital to analog converter (DAC), a 16 channel 12-bit analog 
to digital converter (ADC), 8 digital $\mathrm{H} / \mathrm{O}$ lines, 16-bit direct memory access (DMA), a $1.2 \mathrm{~Gb}$ hard disc, and a $150 \mathrm{Mb}$ magnetic tape drive. The DAC generates the analog ramp waveform to drive the piezoelectric drivers, and the ADC's digitize the spectral intensities from the FPI. The I/O lines are the output from the system to enable control action in the event that a redline has been encountered. The hard disc is used for data storage during engine firing, and the DMA enables high speed writing to the disc. The magnetic tape unit is used for archive purposes.

\section{SUMMARY}

The overall objective of this effort is to develop instrumentation that enables spectroscopic measurement of eroding engine components during fight. This instrumentation consists of a nozzle mounted optic, an FPI spectrometer, and a fiberoptic cable to transmit the collected spectral radiation from the optic to the spectrometer. These devices are currently being fabricated, and scheduled for testing on the TTB engine at MSFC.

Nozzle mounted optics observe spectral phenomena generated within the plume of a rocket engine during flight. Three types of nozzle mounted optic probes for application on the SSME are being developed. These are a combustion chamber emission viewing optic, a Mach disc emission viewing optic, and an exit plane absorption optic. The chamber view optic has been tested on the TTB engine. Structurally it survived 10 engine firings, after which it failed due to gas impingement. Spectroscopically it was found that emissions from the SSME combustion chamber appear similar to a black body with the peak at $520 \mathrm{~nm}$. Upon conclusion of testing the three probes, one will be selected for flight application.

For installation of the nozzle mounted optic onto the SSME, no modifications to the engine are required. Analysis has shown that the optic will be capable of viewing the plume throughout the entire flight envelope. Testing has indicated that the device will withstand the thermal and mechanical loads imposed by engine operation. The optic assembly poses no risk to the engine based on analysis performed to date.

The FPI spectrometer is a rugged, lightweight, compact, high resolution spectrometer capable of detecting the spectral signatures of eroding engine components. This spectrometer is smaller and lighter than conventional grating or prism devices, and will provide greater wavelength resolving capability. A three channel breadboard FPI spectrometer was fabricated and tested; demonstrating the resolution, sensitivity, signal to noise, and environmental insensitivity desired for flight compatible spectrometers. Within the limitations inherent in the breadboard design, the key features required to provide confidence in the FPI have been demonstrated.
The 8 channel unit that is currently being fabricated has real time digital processing capabilities $(10 \mathrm{~ms})$ with active background discrimination. The device will have a bandwidth of 320 to $430 \mathrm{~nm}$ and a resolution of $0.05 \mathrm{~nm}$. This unit will monitor eight spectral lines corresponding to eight distinct metallic species. These species include chromium, cobalt, iron, nickel, manganese, tungsten, calcium, and copper. It has been shown that these species will enable observation of bearing degradation and erosion of any alloy from components along the propellant flow paths.

\section{REFERENCES}

1. "Office of Space Flight Technology Requirements," National Aeronautics and Space Administration Office of Space Flight, Washington, D.C., 4/91.

2. Cikanek, H.A., et al., "Space Shuttle Main Engine Plume Spectral Monitoring Preliminary Results", AIAA 87$1792,7 / 87$.

3. Powers, W.T. and Cikanek, H.A., "Analysis of UV-VIS Spectral Radiation from SSMEPlume", Advanced Earth to Orbit Propulsion Technology 1988, Vol. 2, R.J. Richmond and S.T. Wu, eds., NASA CP-30120-VOL-2, 1988, pp.595-611.

4. Wallace, T.L., and Cooper, A.E., "Preliminary Analysis of SSME Baseline Plume Emissions", 2nd Annual Health Monitoring Conference for Space Propulsion Systems, Univ. of Cincinnati, 1990, pp 142-159.

5. SSME Engineering, "SSME Structural Loads Criteria", RSS-8561-24, Rockwell International, 1/91.

6. Nickerson, G., et al., "The Two Dimensional Kinetic (TDK) Reference Computer Program", Engineering and Programming Manual, Software Engineering Associates, Inc., prepared for Contract NAS8-35931, NASA MSFC, $4 / 85$.

7. Powers, W.T., et al., "OPAD Through 1991 Status Report \#2", 3rd Annual Health Monitoring Conference for Space Propulsion Systems, University of Cincinnati, 1991, pp 169-184.

8. Tejwani, G.D. and Gardner, D.G., "Preliminary Report: Engine Diagnostics Database Development and DTF Seeding Experiment Test Matrix", NASA-SSC Report No. TWR J9-A901, 7/89.

9. Bickford, R.L., and Madzsar, G.C., "Fabry-Perot Interferometer Development for Rocket Engine Plume Spectroscopy", AIAA paper 90-2234, 7/90.

10. Madzsar, G.C., "Determination of Alloy Content From Plume Spectral Measurements", AIAA 91-2531, 6/91

11. Reese, G., et al., "Detection and Identification of Alloys In Rocket Engine Exhaust Plumes", 4th Annual Health Monitoring Conference for Space Propulsion Systems, University of Cincinnati, 1992, pp 159-178. 
- Early Warning of Impending Failure

Diagnosis of Component Degradation

Inspection and Maintenance Planning

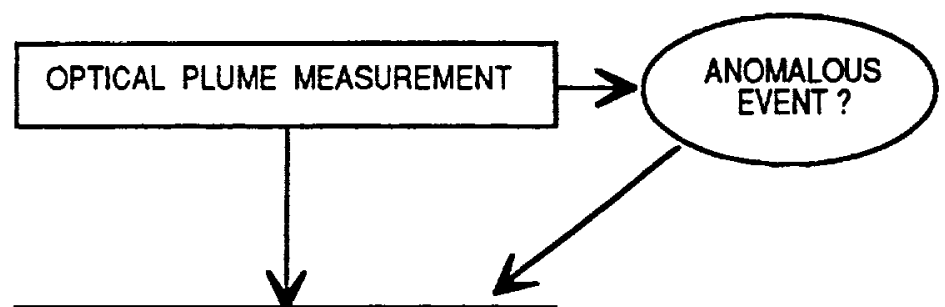

Failure Analysis and Return to Flight

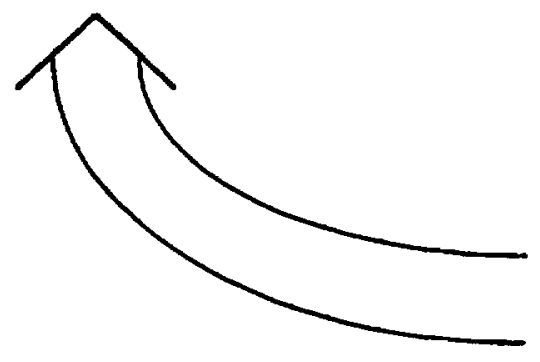

DETERMINATION OF OBSERVABLES

- Material(s), alloy(s) composition

- Erosion rales (grams/sec)

CUMULATIVE ENGINE

DATABASE

ASSESSMENT OF ENGINE HEALTH

- Component Erosion

- Precursor Recognition

- Time History of Erosion

Figure 1.-Methodology for determining engine health from plume emissions.

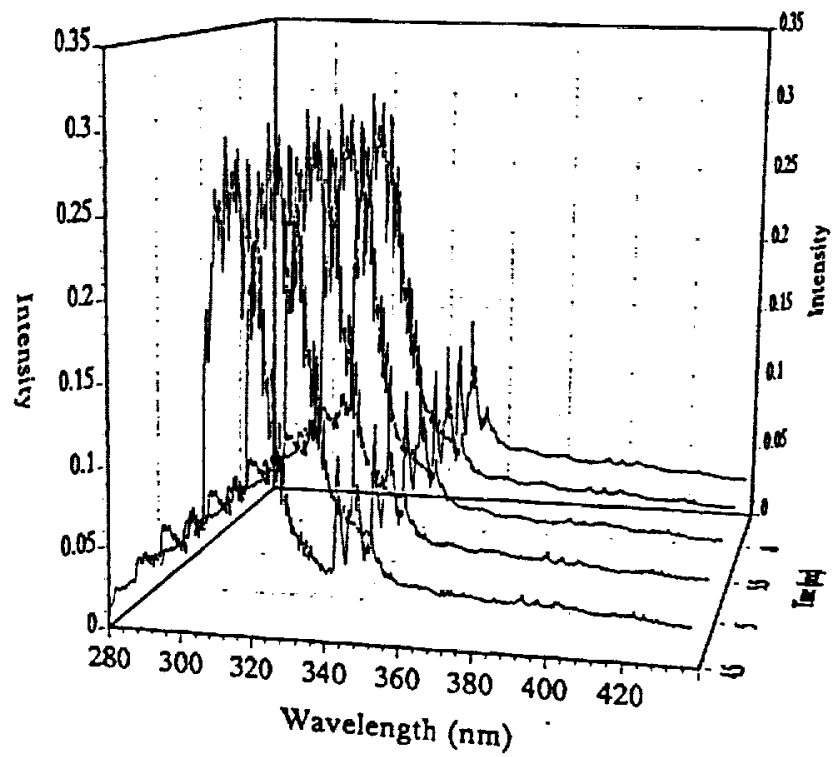

Figure 2.-Emission spectra from nominal SSME firing.

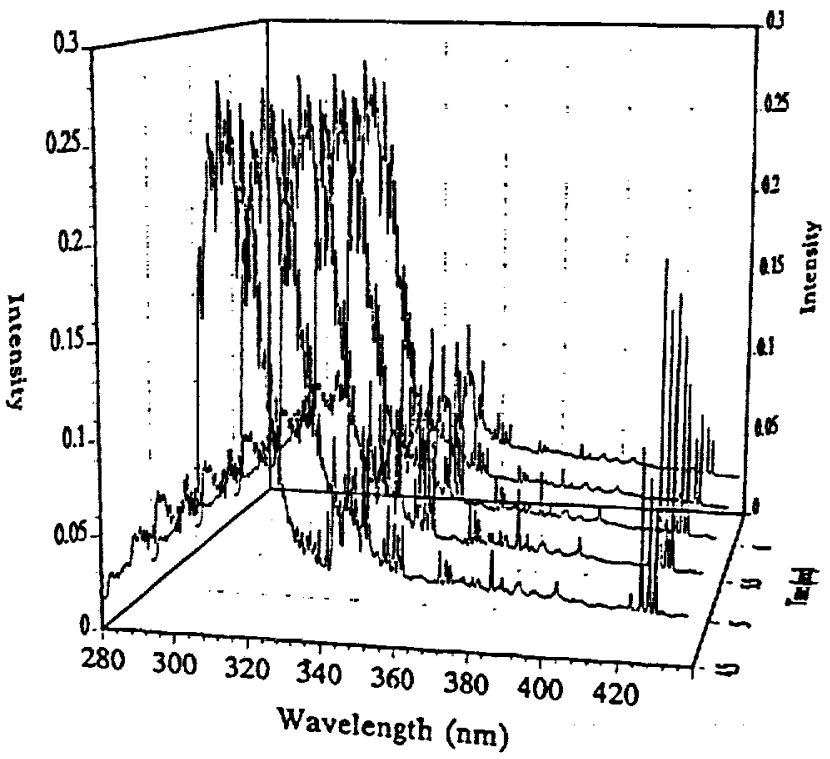

Figure 3-Emission spectra from SSME during degradation. 


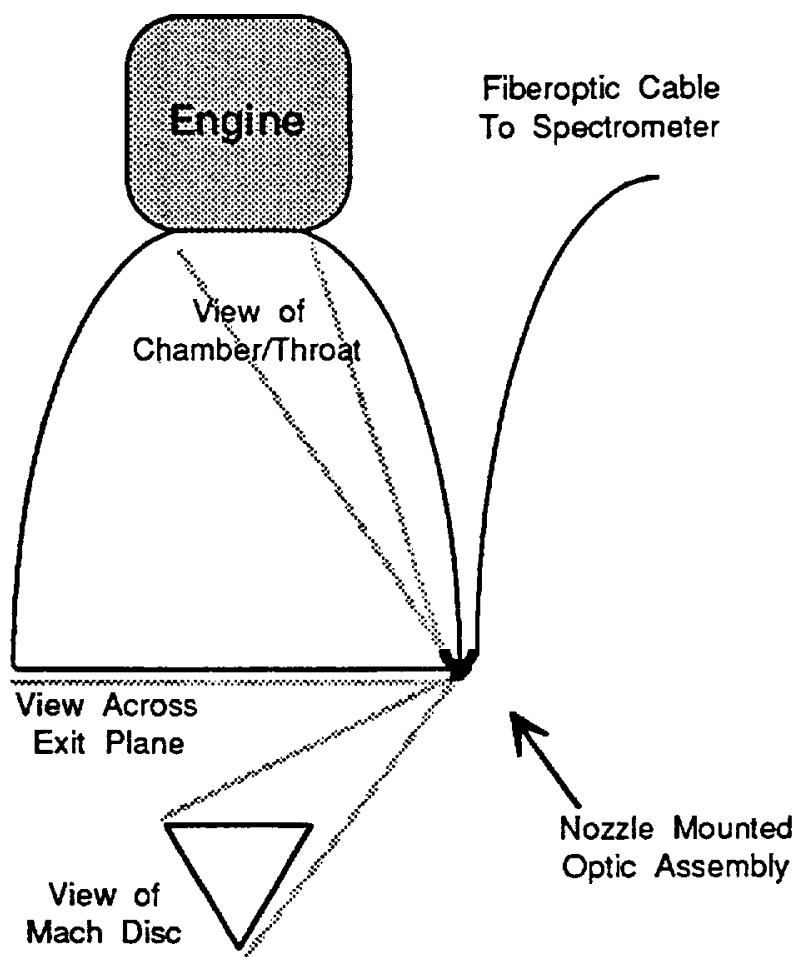

Figure 4.-Optical access to engine exhaust plume.

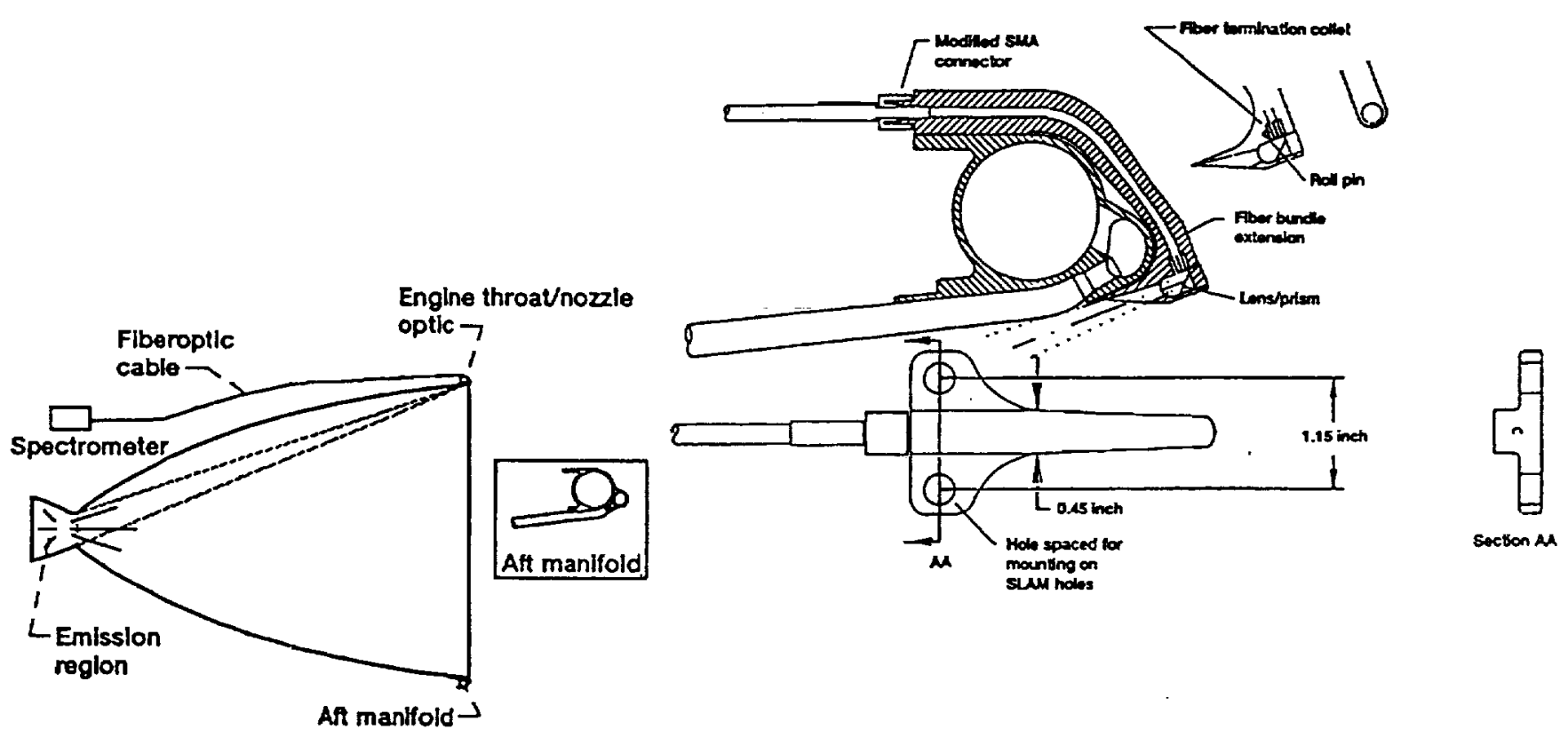

Figure 5.-Nozzle mount optic for viewing the throat and combustion chamber of the engine. 


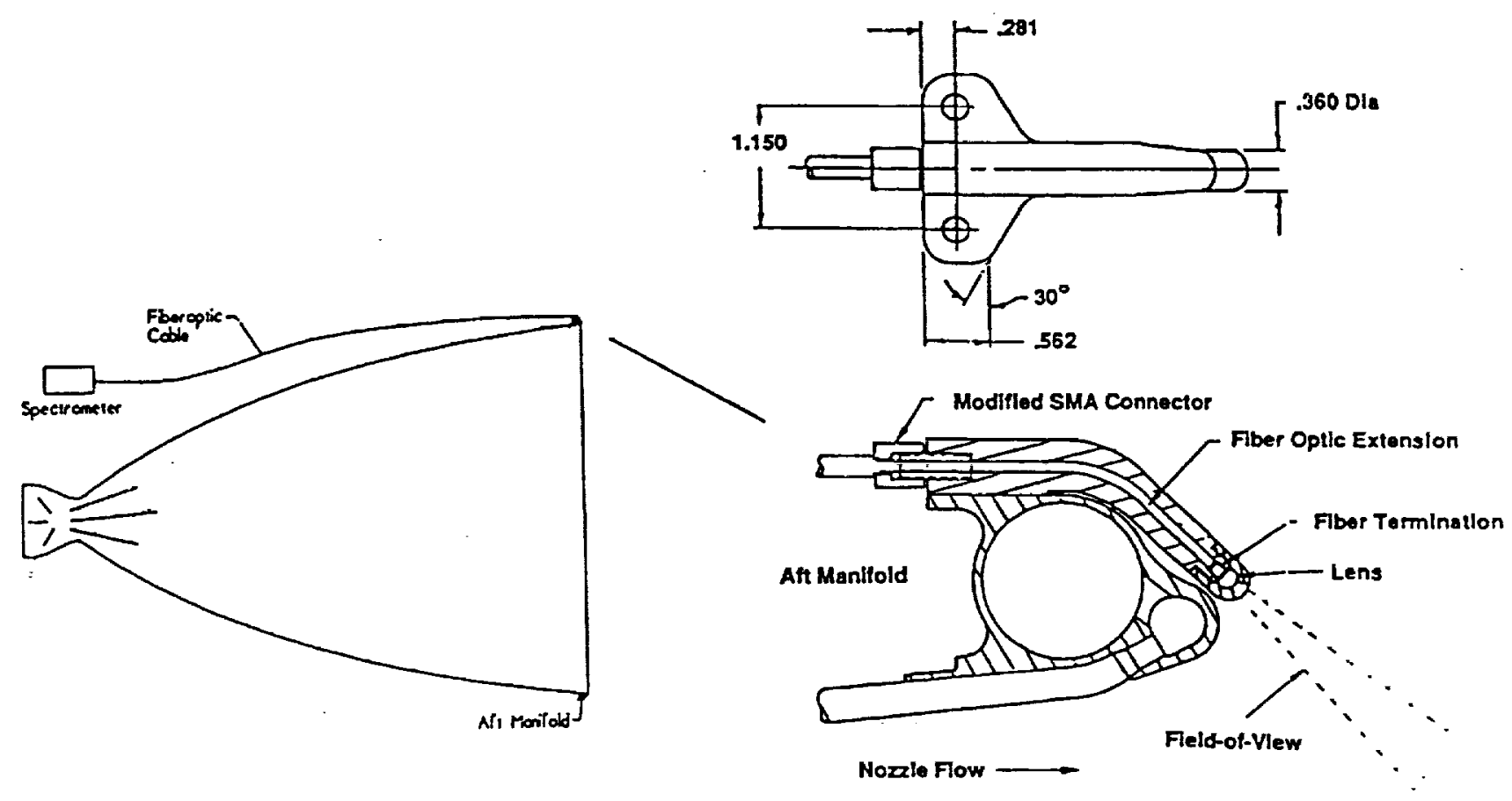

Figure 6.-Nozzle mounted optic for viewing the Mach disc.
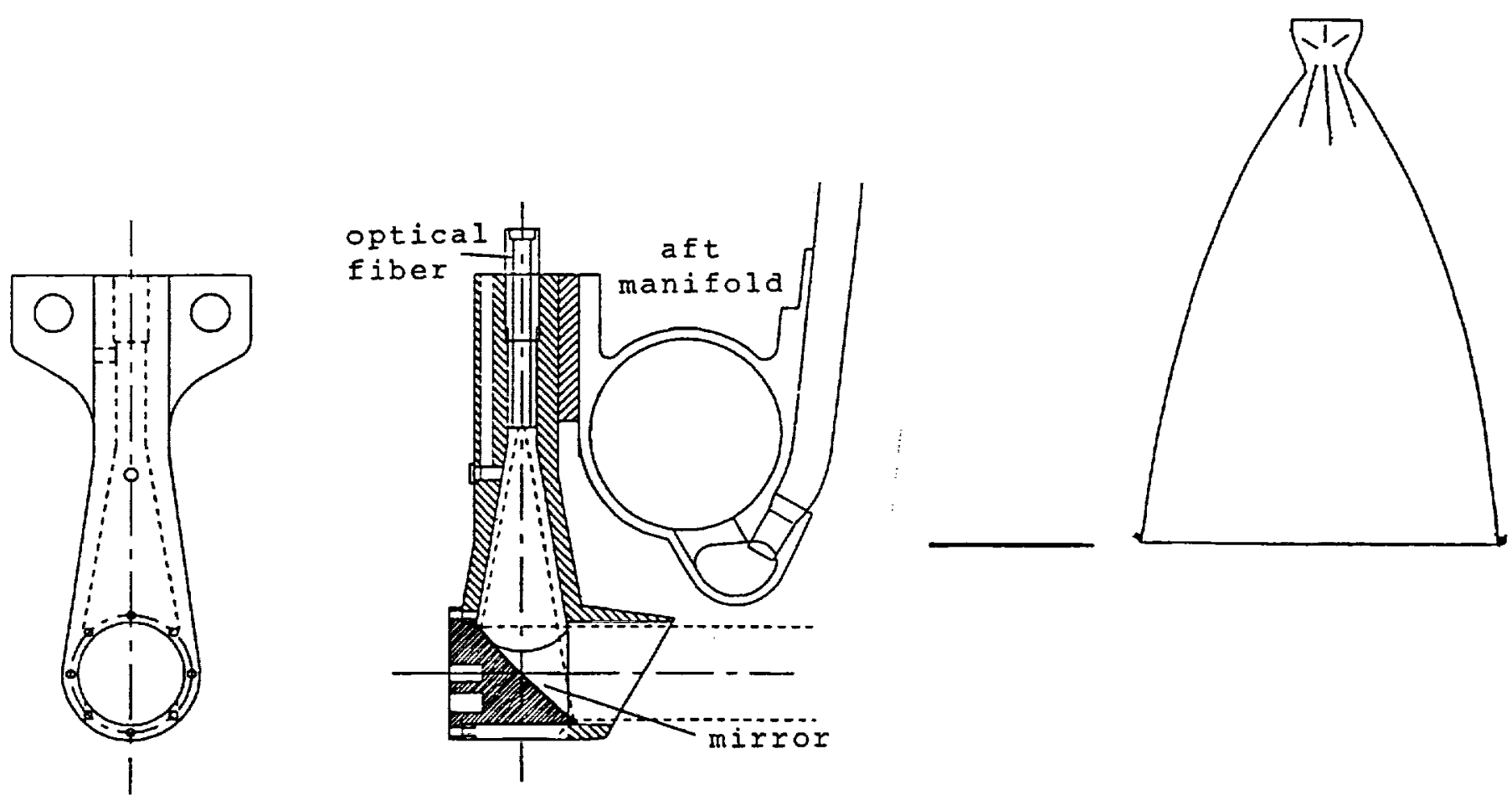

Figure 7.-Nozzle mounted absorption optic. 

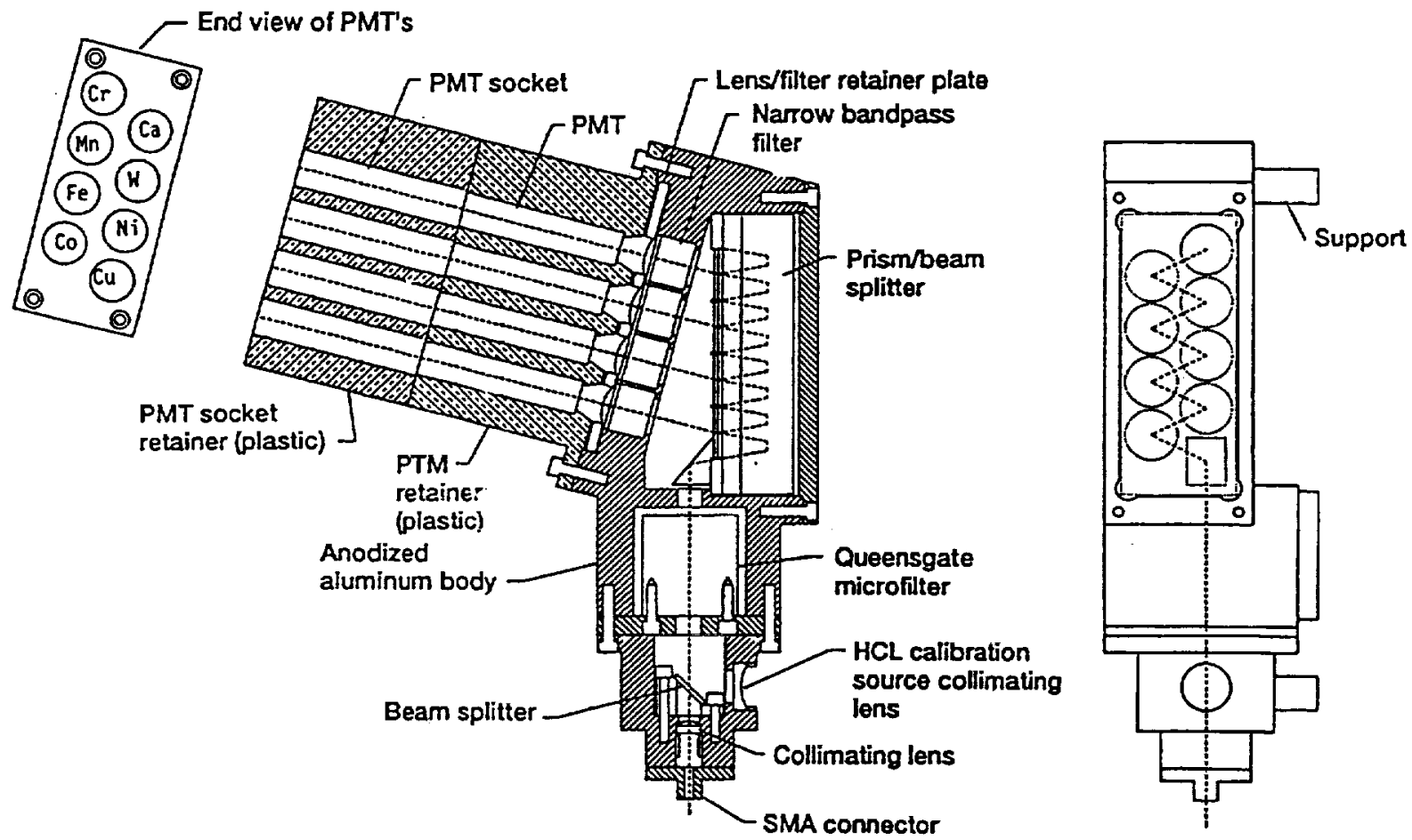

Figure 8.-Schematic of the 8 channel Fabry-Perot interferometric spectrometer. 
Public reporting burden for this collection of information is estimated to average 1 hour per response, including the time for reviewing instructions, searching existing data sources, gathering and maintaining the data needed, and completing and reviewing the collection of information. Send comments regarding this burden estimate or any other aspect of this collection of information, including suggestions for reducing this burden, to Washington Headquarters Services, Directorate for Information Operations and Reports, 1215 Jefferson Davis Highway. Suite 1204, Artington, VA 22202-4302, and to the Office of Management and Budget, Paperwork Reduction Project (0704-0188), Washington, DC 20503.

\begin{tabular}{|l|c|c|}
\hline 1. AGENCY USE ONLY (Leave blank) & $\begin{array}{c}\text { 2. REPORT DATE } \\
\text { June } 1994\end{array}$ & $\begin{array}{r}\text { 3. REPOAT TYPE AND DATES COVERED } \\
\text { Technical Memorandum }\end{array}$
\end{tabular}

\section{TITLE AND SUBTITLE}

Instrumentation for In-Flight SSME Rocket Engine Plume Spectroscopy

George C. Madzsar, Randall L. Bickford, and David B. Duncan
5. FUNDING NUMBERS

WU-323-01-OB

8. PERFORMING ORGANIZATION REPORT NUMBER

E-8911
Cleveland, Ohio 44135-3191

9. SPONSORING/MONITORING AGENCY NAME(S) AND ADDRESS(ES)

National Aeronautics and Space Administration

Washington, D.C. 20546-0001
10. SPONSORING/MONITORING AGENCY REPORT NUMBER

NASA TM-106596

AIAA-94-2985

\section{SUPPLEMENTARY NOTES}

Prepared for the 30th Joint Propulsion Conference cosponsored by AIAA, ASME, SAE, and ASEE, Indianapolis, Indiana, June 27-29, 1994. George C. Madzsar, NASA Lewis Research Center; Randall L. Bickford, Aerojet Propulsion Division, Sacramento, California 95813 (work funded by NASA Contract NAS3-25883); and David B. Duncan, Duncan Technologies, Auburn, California, 95603 (work funded by NASA Contract NAS3-25883). Responsible person, George C. Madzsar, organization code 5310, (216) 433-7434.

12a. DISTRIBUTIONAVAILABILITY STATEMENT

12b. DISTRIBUTION CODE

Unclassified - Unlimited

Subject Category 35

13. ABSTRACT (Maximum 200 words)

This paper describes instrumentation that is under development for an in-flight demonstration of a plume spectroscopy system on the space shuttle main engine. The instrumentation consists of a nozzle mounted optical probe for observation of the plume, and a spectrometer for identification and quantification of plume content. This instrumentation, which is intended for use as a diagnostic tool to detect wear and incipient failure in rocket engines, will be validated by a hardware demonstration on the Technology Test Bed engine at the Marshall Space Flight Center.

14. SUBJECT TERMS

Spectroscopy; Rocket engine; Health monitoring

16. PRICE CODE

$$
\mathrm{A03}
$$

\begin{tabular}{|c|c|}
\hline $\begin{array}{c}\text { 17. SECURITY CLASSIFICATION } \\
\text { OF REPORT } \\
\text { Unclassified }\end{array}$ & $\begin{array}{c}\text { 18. SECURITY CLASSIFICATION } \\
\text { OF THIS PAGE } \\
\text { Unclassified }\end{array}$ \\
\hline
\end{tabular}
19. SECURITY CLASSIFICATION OF ABSTRACT Unclassified

